03

\title{
Комбинированное энергосиловое воздействие на источник в режиме постоянного числа Маха с заданной внешней силой
}

\section{(C) А.Н. Кучеров}

Центральный аэрогидродинамический институт им. проф. Н.Е. Жуковского, 140180 Жуковский, Московская область, Россия

e-mail: ank@aerocentr.msk.su, ank19512006@rambler.ru

\section{(Поступило в Редакцию 21 апреля 2016 г.)}

Изучено комбинированное воздействие на источник, истекающий в затопленное пространство или в вакуум в режиме постоянного числа Маха. Воздействие внешней силой задано с постоянной функцией распределения (сила задана на единицу объема) и с функцией распределения, пропорциональной плотности газа (сила задана на единицу массы). Исследования выполнены для цилиндрического и сферического источников. Проанализированы сходство и различия, преимущества и недостатки вышеперечисленных случаев и вариантов. Показано значительное увеличение энтальпии в дозвуковом потоке (при числе Маха меньше единицы), в цилиндрическом источнике в несколько раз и в сферическом источнике более чем в десять раз. Прирост полной энтальпии увеличивается с ростом протяженности зоны воздействия, с увеличением координаты замыкающего сечения.

DOI: $10.21883 /$ JTF.2017.02.44123.1863

\section{Введение}

Возможные режимы течений в источнике, стоке, вихреисточнике, вихрестоке: дозвуковые в затопленное пространство и из затопленного пространства и два сверхзвуковых, в вакуум и из разреженного пространства (из вакуума) [1-3]. В невозмущенном вихреисточнике (стоке) есть минимальный радиус, на котором радиальное число Маха равно единице. От этого сечения начинаются (или в нем заканчиваются) дозвуковая и сверхзвуковая ветви решения, описывающие четыре режима вихреисточника (стока).

Энергоподвод или внешняя сила могут изменить число Маха так, что на расстояниях больше минимального радиуса радиальное число Маха обращается в единицу. Стационарный расход не может выполниться, наступает кризис аналогично тому, как это происходит в строго одномерном потоке с параллельными линиями тока $[4,5]$. Исследование течений вихреисточника с энергообменом и (или) воздействием внешней силой $[6,7]$ показывает, что достижение радиальным числом Маха значения единица возможно как за счет энергоподвода (нагрева), так и за счет теплоотвода (охлаждения) [8], за счет ускоряющего действия внешней силы [9], или тормозящего (сила направлена против потока).

Проблема управления течением вихреисточника, источника (стока) включает режимы с постоянным числом Maха [10]. Анализ показывает, что раздельное применение энергетического и силового механизмов $[6,7]$ воздействия на источник (сток) газа с расходом $m$, с интенсивностью энергоподвода $g(r)$ или интенсивностью внешней силы $F(r)$ допускает четыре ситуации поддерживания числа Маха постоянным:
1) $m>0$, источник, $g(r)>0$, энергоподвод (нагрев), внешняя сила равна нулю $F(r)=0$;

2) $m>0$, источник, $g(r)=0$, энергоподвода нет, сила $F(r)<0$, торможение газа;

3) $m<0$, сток, $g(r)=0$, без энергообмена, сила $F(r)<0$, ускорение потока;

4) $m<0$, сток, $g(r)<0$, охлаждение (энергоотвод), сила отсутствует $F(r)=0$.

В ситуациях 1 и 4 температура $T$, модуль скорости $u$ (направление скорости указывает знак расхода $m$ ), полная энтальпия $H$ возрастают с ростом координаты $r$, плотность $\rho$ и давление $p$ - убывают. В ситуациях 2 и 3 все перечисленные газодинамические величины возрастают с уменьшением координаты.

Комбинированное использование энергоподвода и силового воздействия может расширить возможности управления параметрами источника и вместе с тем усложнить анализ течения, реализацию в эксперименте и приложениях. Комбинированное применение энергоподвода и внешнего силового воздействия в настоящей работе рассматривается для источника $(m>0)$, с постоянным распределением внешней силы $F(r)=$ const (заданным на единицу объема) или пропорциональным плотности газа $F(r)=$ const $\times \rho(r)$ (заданным на единицу массы). Распределение энергоподвода $g(r)$ подбирается из условия постоянства числа Маха. Рассмотрим цилиндрический и сферический источники. Особое внимание уделим изменениям полной энтальпии вследствие воздействия силой и энергоподводом, рассмотрим также ориентировочную границу (по параметрам подобия) уменьшения энтальпии до нуля. Исследуем зависимости основных характеристик от параметров подобия. 


\section{1. Постановка задачи}

В общем случае управление источником описывается системой безразмерных уравнений сохранения массы, импульса, энергии (1)-(3) и уравнением состояния газа (5) [8-10]:

$$
\begin{aligned}
& \frac{1}{u} \frac{d u}{d r}+\frac{1}{\rho} \frac{d \rho}{d r}+\frac{n}{r}+0 \\
& r^{n} \rho u=m \equiv \frac{m_{0}}{2^{n} \pi \rho_{0} u_{0} r_{0}^{n}}, \\
& u \frac{d u}{d r}+\frac{\gamma-1}{2 \gamma \rho} \frac{d p}{d r}=\frac{F(r)}{2 \rho}, \\
& F(r)=\left\{\begin{array}{l}
\rho F_{Q} f_{Q}(r) \\
F_{E} f_{E}(r)
\end{array}, F_{E}=\frac{\varphi_{0 E} r_{0}}{2^{n} \pi \rho_{0} h_{0}}, F_{Q}=\frac{\varphi_{0 Q} r_{0}}{2^{n} \pi h_{0}},\right. \\
& \frac{d T}{d r}-\frac{\gamma-1}{\gamma \rho} \frac{d p}{d r}=\frac{g(r)}{\rho u}, \\
& g(r)=\left\{\begin{array}{l}
E g_{E}(r) \\
\rho(r) Q g_{Q}(r)
\end{array}, E=\frac{g_{0 E} r_{0}}{2^{n} \pi \rho_{0} u_{0} h_{0}}, Q=\frac{g_{0 Q} r_{0}}{2^{n} \pi u_{0} h_{0}},\right. \\
& \int_{r_{1}}^{r_{2}} f_{Q}(r) d r=1, \quad \int_{r_{1}}^{r_{2}} g_{E}(r) r^{n} d r=1, \\
& p=\rho T \text {. }
\end{aligned}
$$

В уравнениях (1)-(5) показатель степени равен $n=1$ или 2 для цилиндрического или сферического источника, $m_{0}$ - размерный физический расход, $m-$ соответствующий безразмерный расход, $r_{1}, r_{2}-$ начало и конец зоны воздействия. Характерные величины следующие: минимальный радиус $r_{0}$, на котором в невозмущенном источнике радиальное число Маха равно единице, $M_{r}\left(r_{0}\right)=1$, давление $p_{0}$, температура $T_{0}$, энтальпия $h_{0}=C_{p} T_{0},\left(C_{p}\right.$ - теплоемкость при постоянном давлении), плотность $\rho_{0}-$ в затопленном пространстве, скорость $u_{0}=\sqrt{ }\left(2 h_{0}\right)$. Параметры подобия: показатель адиабаты $\gamma$, силовые параметры $F_{E}, F_{Q}$, которые описываются физическими удельными величинами силы $\varphi_{0 E}, \varphi_{0 Q}$, заданными в единицу объема, $\mathrm{N} / \mathrm{m}^{3}$ или на единицу массы, $\mathrm{N} / \mathrm{kg}$ (приняли $\left.\varphi_{0 E}=\rho_{0} \varphi_{0 Q}\right)$. Энергоподвод и энергетические параметры подобия $E, Q$ задаются удельными величинами энерговыделения $g_{0 E}, g_{0 Q}$ в единицу объема, W/m $\mathrm{m}^{3}$ или на единицу массы, W/kg (приняли $\left.g_{0 E}=\rho_{0} g_{0 Q}\right)$. В настоящей работе энегоподвод $g(r)$ подбирается таким образом, чтобы число Маха $M_{r}$ внутри зоны сохраняло свое значение, равное значению на входе $M_{r}=M_{r 1}=$ const.

Нормировочные интегралы (4) безразмерных функций распределения $f_{Q}(r), f_{E}(r), g_{E}(r), g_{Q}(r)$ берутся при необходимости по модулю. В общем случае нормировку функций $g_{Q}(r), f_{E}(r)$ можно делать без функции плотности $\rho(r)$ под знаком интеграла, как функций $g_{E}(r)$, $f_{Q}(r)$. Результат - изменение энтальпии - можно пересчитывать с учетом найденной плотности $\rho(r)$ так, чтобы силовые и энергетические параметры количественно совпадали с изменением полной энтальпии $\Delta H=H_{2}-H_{1}$ или потока полной энтальпии $m \Delta H$. Уравнение для полной энтальпии $H=T+u^{2}$ следует из уравнений (2), (3):

$$
\frac{d H}{d r}=\frac{g(r)}{\rho u}+\frac{F(r)}{\rho} .
$$

Исследуем ситуации, в которых направление течения совпадает с направлением координаты $r$. Энергоподвод $g(r)$ и внешняя сила $F(r)$ могут быть и положительными и отрицательными. Силу $F(r)$ будем задавать, энергоподвод „сформируем“, используя безразмерное уравнение для числа Маха, так, чтобы получить постоянное значение $M_{r}$, равное $M_{r 1}$ на входе в зону воздействия (здесь и далее нижний индекс „1“ означает „на входе“", „2“ - „на выходе“ $)$ :

$$
\begin{gathered}
\frac{1-M_{r 1}^{2}}{M_{r 1}} \frac{d M_{r}}{d r}=-n \frac{1+M_{r 1}^{2}(\gamma-1) / 2}{r} \\
+\frac{\left(\gamma M_{r 1}^{2}+1\right) g(r)}{2 p u}-\frac{\gamma+1}{\gamma-1} \frac{F(r)}{2 p}=0 .
\end{gathered}
$$

Для искомой функции распределения силы $g(r)$ отсюда следует

$$
\begin{gathered}
\frac{g(r)}{\rho u}=\frac{n T(r)}{D_{1} r T_{1}}+\frac{F(r)}{s D_{1} \rho(r)}, \\
s=2 \frac{\gamma-1}{\gamma+1}, \quad \frac{1}{T_{1}}=1+\frac{\gamma-1}{2} M_{r 1}^{2}, \quad D_{1}=\frac{\gamma M_{r 1}^{2}+1}{2} .
\end{gathered}
$$

Условие постоянного числа Маха дает и связь температуры $T(r)$ со скоростью $u(r)$

$$
\frac{2 u^{2}(r)}{(\gamma-1) T(r)}=M_{r 1}^{2}, \quad T(r)=\frac{2 u^{2}(r)}{(\gamma-1) M_{r 1}^{2}} .
$$

С учетом (9) и начального условия $H_{1}=1=T_{1}+u_{1}^{2}=T_{1}\left[1+(\gamma-1) M_{r 1}^{2} / 2\right]=u_{1}^{2}[1+2 /(\gamma-$ $\left.-1) M_{r 1}^{2}\right]$ интеграл безразмерного уравнения (6) сохранения полной энтальпии $H=T+u^{2}$ есть

$$
\begin{aligned}
H & =T+u^{2}=T(r)\left[1+\frac{\gamma-1}{2} M_{r 1}^{2}\right]=\frac{T(r)}{T_{1}} \\
& =\frac{u^{2}(r)}{u_{1}^{2}}=1+\int_{r_{1}}^{r}\left[\frac{g(r)}{\rho u}+\frac{F(r)}{\rho}\right] d r .
\end{aligned}
$$

В левой части уравнения (10) - полезные связи функций $H, T, u(r)$. Далее из (6) получим решения, описывающие управление источником в режиме постоянного числа Маха. 


\section{2. Комбинированное воздействие при заданных функциях распределения внешней силы $f_{Q}(r)=$ const, $f_{E}(r)=$ const. Уравнения и решения}

Примем функцию распределения внешней силы $F(r)$ в виде

$$
\begin{aligned}
F(r)= & \left\{\begin{array}{l}
F_{E} C_{F E} \\
\rho(r) F_{Q} C_{F Q}
\end{array}, C_{F E}=\frac{1}{r_{2}-r_{1}}=C_{F Q},\right. \\
& f_{E}(r)=C_{F E}, \quad f_{Q}(r)=C_{F Q} .
\end{aligned}
$$

Тогда при поддерживании числа Маха $M_{r 1}$ постоянным, согласно (7), (8), функция энергоподвода имеет следующий вид:

$$
\frac{g(r)}{\rho u}=\frac{n T}{D_{1} T_{1} r}+\frac{1}{s D_{1}}\left\{\begin{array}{l}
F_{E} C_{F E} / \rho(r) \\
F_{Q} C_{F Q} .
\end{array}\right.
$$

\section{1. Сила задана на единицу массы, $F_{Q}$-вариант}

Подстановка выражений (11), (12) в уравнение (6) дает уравнение и решение

$$
\begin{gathered}
\frac{d H}{d x}=\alpha_{2 n} \frac{H(x)}{x}+\alpha_{01}, \quad x=\frac{r}{r_{1}}, \\
\alpha_{01}=F_{Q} C_{F Q} r_{1} s_{2}, \quad \alpha_{2 n}=\frac{n}{D_{1}}, \quad s_{2}=1+\frac{1}{s D_{1}}, \\
H=\left(1-\alpha_{1 n}\right)\left(\frac{r}{r_{1}}\right)^{n / D_{1}}+\alpha_{1 n} \frac{r}{r_{1}}, \\
\alpha_{1 n}=\frac{\alpha_{01}}{1-n / D_{1}} .
\end{gathered}
$$

\section{2. Сила задана в единицу объема, $F_{E}$-вариант}

Уравнение (6) после подстановки $F(r), g(r)$ из (11), (12) имеет вид (15), решение (16):

$$
\begin{gathered}
\frac{d Z}{d x}=\beta_{2 n} \frac{Z(x)}{x}+\beta_{0 n} x^{n} \\
\beta_{0 n}=\frac{F_{E} C_{F E} u_{1} r_{1}^{n+1} s_{2}}{2 m}, \quad \beta_{2 n}=\frac{n}{2 D_{1}} \\
Z=\frac{u(r)}{u_{1}}=\left(1-\beta_{1 n}\right)\left(\frac{r}{r_{1}}\right)^{n / 2 D_{1}}+\beta_{1 n}\left(\frac{r}{r_{1}}\right)^{n+1}, \\
\beta_{1 n}=\frac{\beta_{0 n}}{n+1-n / 2 D_{1}} .
\end{gathered}
$$

Все искомые газодинамические функции связаны между собой благодаря условию постоянного числа Маха
$M_{r}=M_{r 1}=$ const и исходным уравнениям сохранения и состояния газа (1)-(5) (см. также левую часть (10))

$$
\begin{gathered}
H(r)=\frac{T}{T_{1}}=\left(\frac{u(r)}{u_{1}}\right)^{2}, \quad \frac{\rho(r)}{\rho_{1}}=\left(\frac{r}{r_{1}}\right)^{-n}\left(\frac{T}{T_{1}}\right)^{-1 / 2}, \\
\frac{p(r)}{p_{1}}=\left(\frac{r}{r_{1}}\right)^{-n}\left(\frac{T}{T_{1}}\right)^{1 / 2} .
\end{gathered}
$$

При анализе решений внимание обратим, прежде всего, на различия вариантов $n=1$ и 2, цилиндрический и сферический источники, $F_{E}$ и $F_{Q}$-вариантов (сила задана в единицу объема и на единицу массы).

\section{3. Результаты}

\section{1. Распределения газодинамических величин внутри зоны энергосилового воздействия}

На рис. 1, $a$ приведены распределения полной энтальпии $H(r)$ в зоне воздействия при $M_{r 1}=1.5$, в цилиндрическом источнике $(n=1)$ для трех вариантов со значениями силового параметра $F_{Q}=0$ (1), 0.52 (2), -0.52 (3). В последнем случае в замыкающем сечении полная энтальпия $H_{2}=H\left(r_{2}=3\right)$ близка к нулю.

Кривая $1\left(F_{Q}=0\right)$ соответствует чисто энергетическому варианту поддерживания числа Маха постоянным $M_{r 1}=$ const внутри зоны $\left[r_{1}, r_{2}\right]$ за счет подбора функции энергоподвода $g(r)$ согласно $(7),(8)$ :

$$
\begin{gathered}
g(r)=\frac{n m T(r)}{D_{1} T_{1} r^{n+1}}=\frac{m n}{D_{1} r_{1}^{n+1}}\left(\frac{r}{r_{1}}\right)^{n / D_{1}-n-1}, \\
\frac{T(r)}{T_{1}}=\left(\frac{r}{r_{1}}\right)^{n / D_{1}}=H=\frac{u^{2}}{u_{1}^{2}}, \quad \frac{\rho(r)}{\rho_{1}}=\left(\frac{r}{r_{1}}\right)^{-n-n / 2 D_{1}}, \\
\frac{p(r)}{p_{1}}=\left(\frac{r}{r_{1}}\right)^{n / 2 D_{1}-n} .
\end{gathered}
$$

Назовем его базовым. На рис. $1, b$ построены распределения внешней силы $F(r)$, кривые $1-3$ и функции энергоподвода $g(r)$, кривые 4-6 при значениях силового параметра $F_{Q}=0(1), 0.52(2),-0.52$ (3). Вариант с отрицательным силовым параметром $F_{Q}=-0.52$ (торможение) отличается тем, что в этом случае и энергоподвод (кривая 6) является отрицательным (т.е. происходит энергоотвод, охлаждение). Этот факт является следствием выбора функции энергообмена $g(r)$ из условия $M_{r 1}=$ const.

При равном по модулю силовом параметре $F_{Q}=0.52$ полная энтальпия в конце зоны комбинированного энергосилового воздействия увеличилась более чем в 3 раза. Энергоподвод $g(r)$, кривая 5 , по всей зоне положительный, как и в базовом варианте, кривая 4.

Аналогичный анализ выполнен для варианта сферического источника $(n=2)$ при $F_{Q}=0,0.69,-0.69$; последний вариант соответствует энтальпии $H_{2}=H\left(r_{2}=3\right)$, близкой к нулю (рис. 2, , кривая 2). Значение полной 

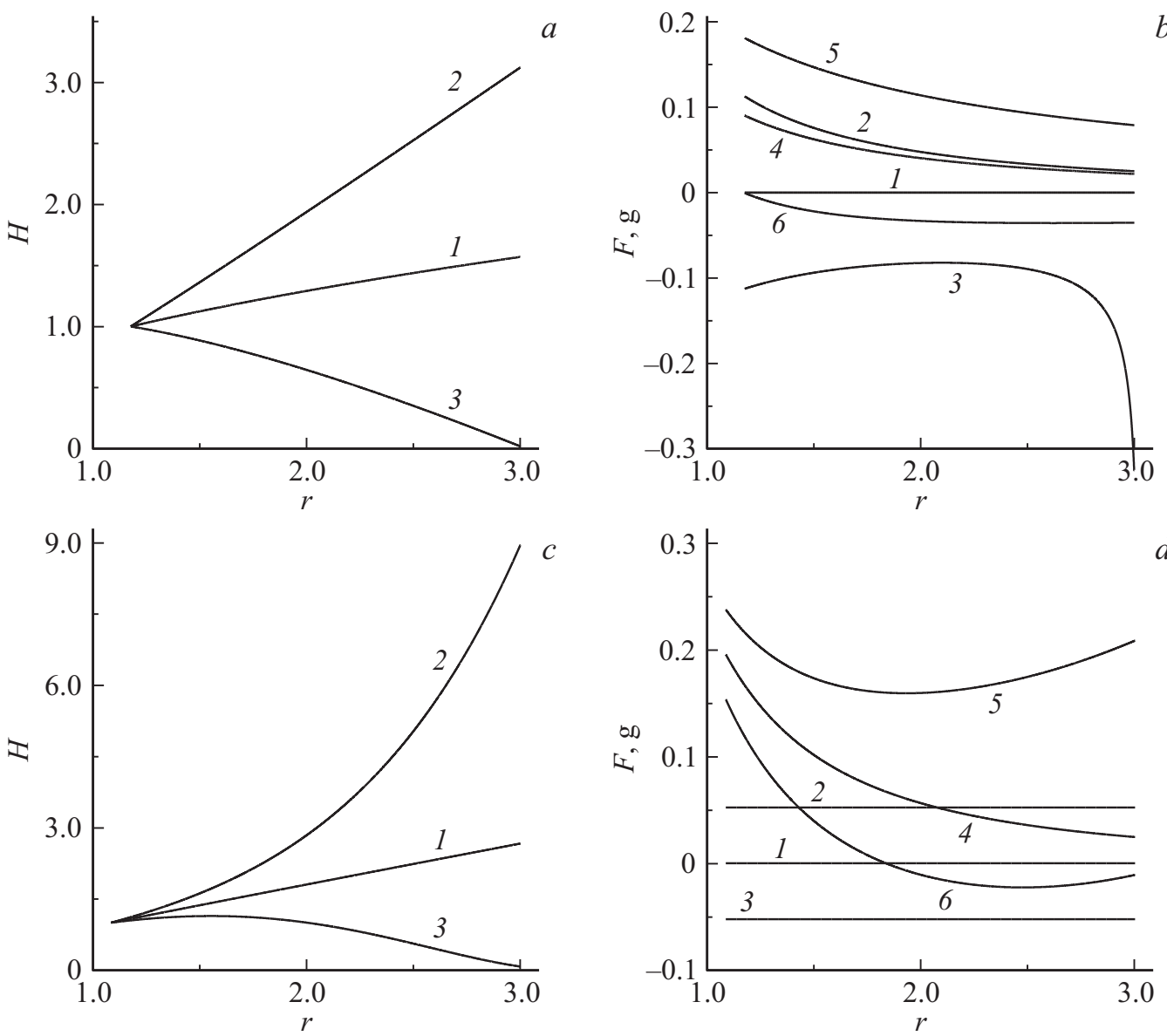

Рис. 1. $a-$ полная энтальпия $H(r)$ в цилиндрическом источнике $(n=1)$ с числом Маха $M_{r 1}=1.5$ при комбинированном воздействии: $1-F_{Q}=0,2-0.52,3--0.52 ; r_{2}=3 ; b-$ внешняя сила $F(r)=\rho(r) F_{Q} C_{Q}$, кривые $1-3\left(F_{Q}=0,0.52,-0.52\right)$; энергоподвод $g(r)$, кривые $4-6\left(F_{Q}=0,0.52,-0.52\right) ; c-$ сферический источник $(n=2)$, полная энтальпия $H(r): 1-F_{E}=0$, $2-0.1,3--0.1 ; r_{2}=3, M_{r 1}=1.5 ; d-F(r)=F_{E} C_{E}$, кривые $1-3\left(F_{E}=0,0.1,-0.1\right) ; g(r)$, кривые $4-6\left(F_{E}=0,0.1,-0.1\right)$.

энтальпии в варианте $F_{Q}=0.69$ в конце зоны воздействия превысило первоначальное значение более чем в 5 раз. Функция энергоподвода $g(r)$ положительная, кривая 5 , как и в базовом варианте, кривая 4 . В варианте $F_{Q}=-0.69$ функция энергоподвода $g(r)$ на завершающем участке, протяженностью около двух третей от полной длины зоны, отрицательная (охлаждение). Функция распределения внешней силы $F(r)$ при отрицательных значениях силового параметра $F_{Q}$, как в цилиндрическом источнике $(n=1)$, так и в сферическом $(n=2)$ имеет максимум во второй половине зоны и минимумы в начале и в конце (рис. $1, b)$.

На рис. $1, c, d$ рассматриваются ситуации с заданными значениями силового параметра $F_{E}=0$ (кривая 1 ), $0.1(2),-0.1$ (3) для сферического источника и обсуждаются варианты при $F_{E}=0,0.2,-0.2$ для цилиндрического источника. В последнем случае при $F_{E}=-0.2$ полная энтальпия в конце зоны $H_{2}=H\left(r_{2}=3\right)$ близка к нулю; в сферическом источнике, как видим на рис. 1, $c$, при $F_{E}=-0.1$ полная энтальпия $H_{2}$ также близка к нулю.

Увеличение энтальпии $H_{2}$ в конце зоны при $F_{E}=0.1$ почти девятикратное в сферическом источнике (рис. 1, $c$, кривая 2) и шестикратное в цилиндрическом источнике.
Сопоставление распределений внешней силы $F(r)$ для отрицательных значений силового параметра $\left(F_{E}=-0.1 ; F(r), g(r)\right.$ - кривые 3,6 на рис. $\left.1, d\right)$ в случаях сферического $(n=2)$ и цилиндрического источника $\left(n=1, F_{E}=-0.2\right.$, графики $F(r), g(r)$ аналогичны кривым 3,6 на рис. $1, b)$ показало, что, как и в $F_{Q^{-}}$ вариантах, функция энергоподвода $g(r)$ полностью отрицательна (охлаждение) в цилиндрическом источнике и отрицательна во второй половине зоны в сферическом источнике (кривая 6 , рис. $1, d$ ).

Строго говоря, предел значений силового параметра $F_{Q 0}$ или $F_{E 0}$, при котором энтальпия в замыкающем сечении обращается в нуль, составляет согласно решениям $H\left(r_{2}\right)=0$ :

$$
\begin{gathered}
F_{Q 0}=\frac{1}{\left(1-1 / A_{1}\right) A_{0}}, A_{0}=\frac{C_{Q} s_{2} r_{1}}{1-n / D_{1}}, A_{1}=\left(\frac{r_{2}}{r_{1}}\right)^{n / D_{1}-1}, \\
F_{E 0}=\frac{1}{\left(1-1 / B_{1}\right) B_{0}}, \quad B_{0}=\frac{C_{E} s_{2} u_{1} r_{1}^{n+1}}{n+1-n / 2 D_{1}}, \\
B_{1}=\left(\frac{r_{2}}{r_{1}}\right)^{n / 2 D_{1}-1-n} .
\end{gathered}
$$



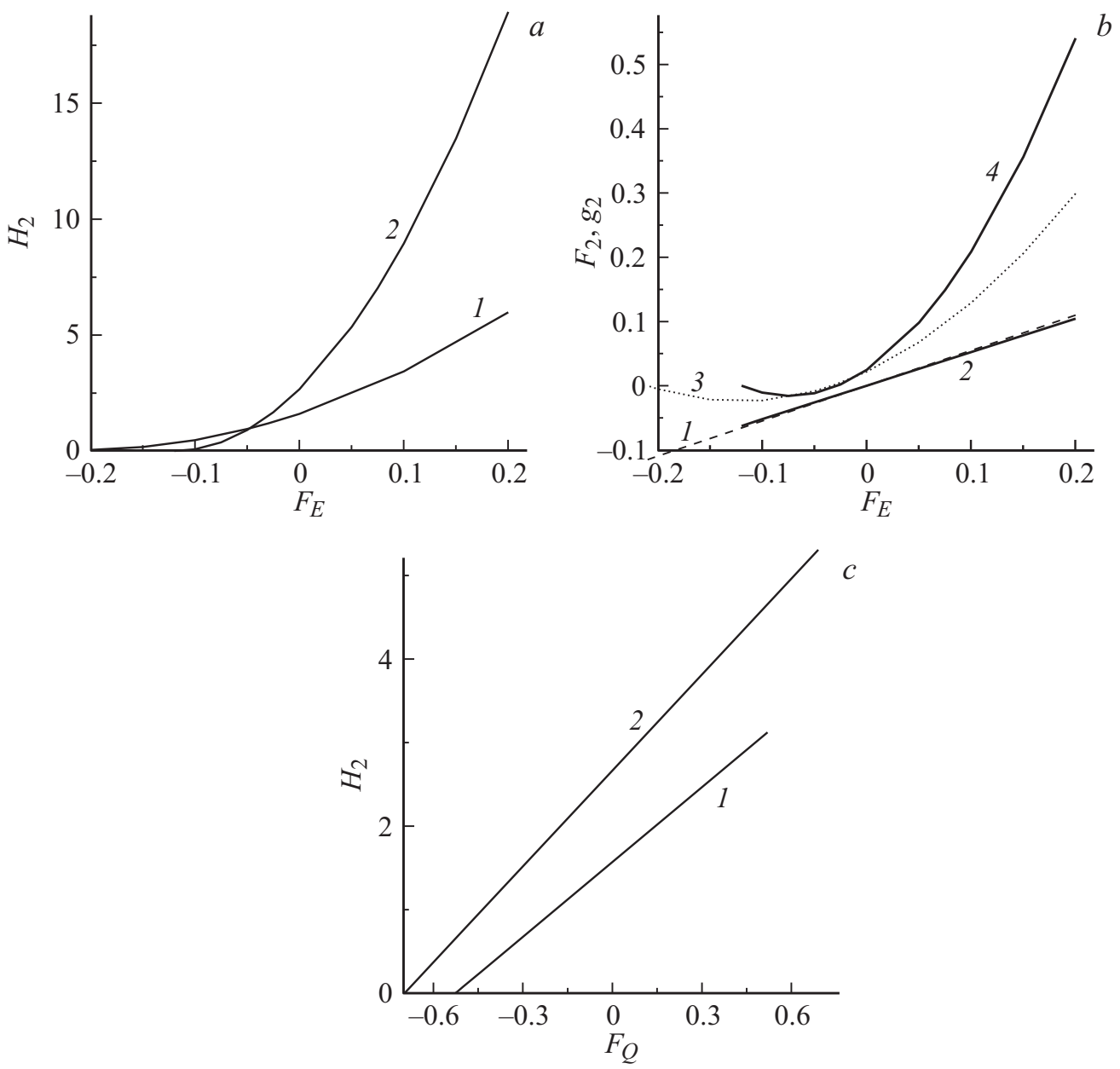

Рис. 2. $a$ - энтальпия $H_{2}=H\left(r_{2}\right)$ как функция силового параметра $F_{E}$ в конце зоны $r_{2}=3, M_{r 1}=1.5: 1-$ цилиндрический источник, $n=1,2-$ сферический, $n=2 ; b-$ внешняя сила $F_{2}=F\left(r_{2}\right)=F_{E} C_{F E}$ как функция силового параметра $F_{E}$, кривые 1,2 $(n=1$ и 2$)$; энергоподвод $g_{2}=g\left(r_{2}\right)$, кривые $3,4\left(n=1\right.$ и 2); $c$ - энтальпия $H_{2}$ как функция силового параметра $F_{Q}$, кривые 1,2 $(n=1$ и 2$)$.

Для примеров, рассмотренных выше, значения силового параметра $F_{Q 0}$ составили $F_{Q 0} \approx 0.5261(n=1)$, $-0.6967(n=2)$, силового параметра $F_{E 0} \approx-0.2101$ $(n=1),-0.1200(n=2)$.

При некоторых достаточно малых значениях температуры газа $T_{2}$ модель совершенного газа несправедлива, например, для воздуха, рассматриваемого как смесь азота и кислорода (температура перехода в жидкое состояние 70 и $90 \mathrm{~K}$ ) [11-13]. Аналогично при достаточно больших температурах, например для воздуха свыше $1000 \mathrm{~K}$, модель совершенного газа требует поправок, в первую очередь, для теплоемкости.

\section{2. Полная энтальпия $\mathrm{H}_{2}$ за зоной как функция силового параметра $F_{Q}, F_{E}$}

Итоговые изменения полной энтальпии к концу зоны $H_{2}=H\left(r_{2}\right)$ сопоставим при различных значениях силовых параметров $F_{Q}, F_{E}$.

На рис. 2, $a$ собраны зависимости полной энтальпии $H_{2}=H\left(r_{2}\right)$ в конце зоны от силового параметра $F_{E}$ в диапазоне $(-0.2,0.2)$ для цилиндрического источника, кривая $1, n=1$, и для сферического источника, кривая 2 , $n=2$. Такие изменения энтальпии $\mathrm{H}_{2}$ обусловлены внешней силой $F(r)\left(F_{2}=F\left(r_{2}\right)\right.$, кривые 1,2 на рис. $2, b$ как функции от силового параметра $\left.F_{E}\right)$ и энергоподводом $g(r)$ (кривые $3,4, g_{2}=g\left(r_{2}\right)$ ). Отметим, что функции распределения внешней силы $F(r)$ совпадают, с небольшим отклонением, на общем интервале значений силового параметра $F_{2}=F\left(r_{2}\right)$ в диапазоне $F_{E}$ от -0.12 до 0.2 (см. кривые 1,2 ) для цилиндрического и сферического источников. Функции энегоподвода $g_{2}=g\left(r_{2}\right)$ в конце зоны при изменении силового параметра от значений -0.075 до 0 также близки при $n=1$ и 2 (цилиндрический источник, кривая 3 ; сферический источник, кривая 4). С ростом $F_{E}$, например до значений $0.1,0.2$, величина $g_{2}$ для сферического источника проходит заметно выше, чем для цилиндрического в режиме постоянного числа Маха $M_{r 1}=$ const $\left(=M_{r 1}=1.5\right.$ в данном случае).

На рис. 2, $c$ приведены зависимости энтальпии $\mathrm{H}_{2}$ от силового параметра $F_{Q}$, аналогичные зависимостям 

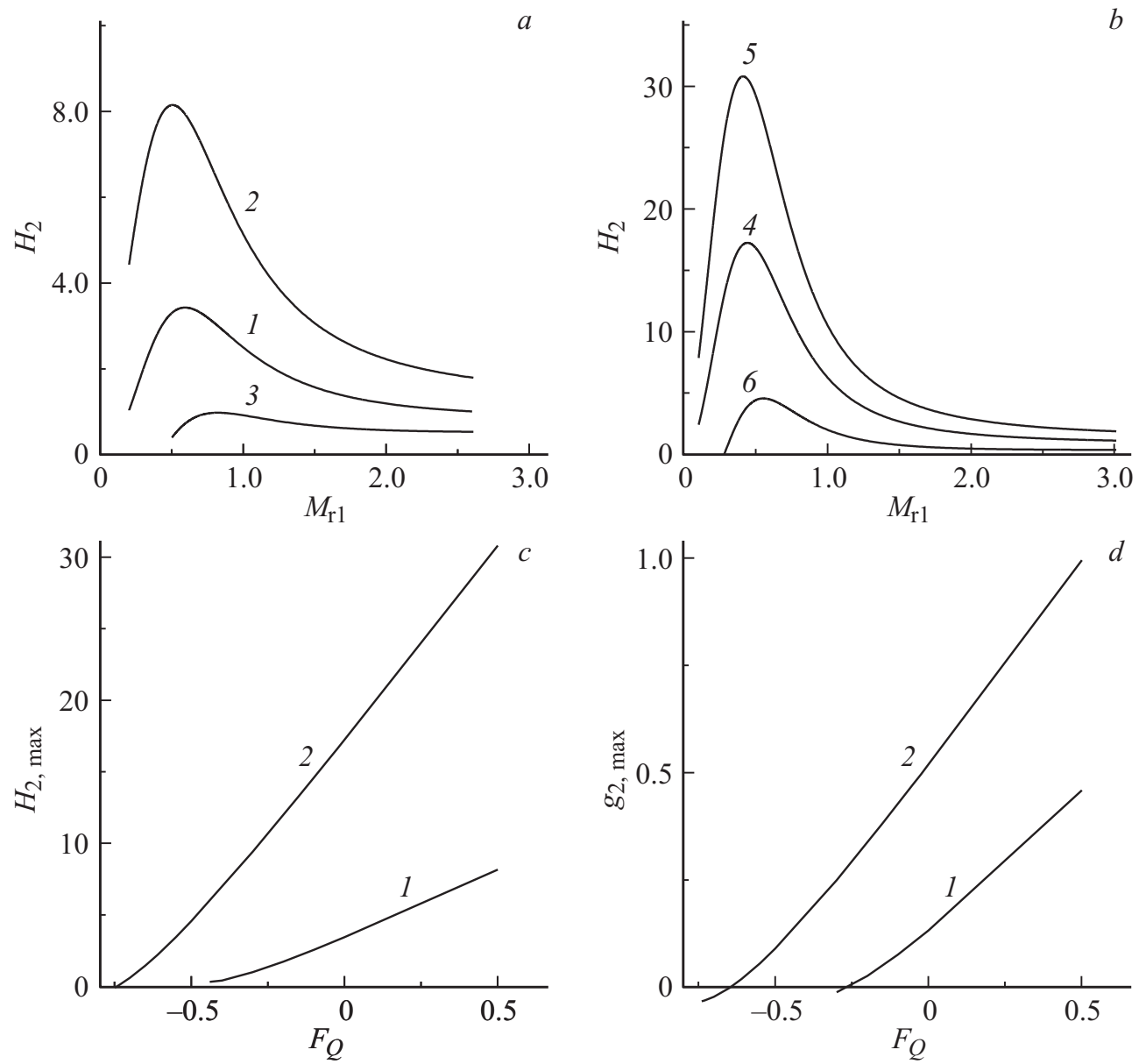

Рис. 3. $a-$ энтальпия на выходе $H_{2}$ как функция числа Маха на входе $M_{r 1}: 1-F_{Q}=0,2-0.5,3--0.3$, цилиндрический источник $n=1$, замыкающая координата $r_{2}=3 ; b-$ энтальпия $H_{2}$ как функция $M_{r 1}: 4-F_{Q}=0,5-0.5,6--0.5$, сферический

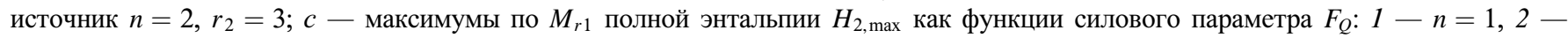
$n=2 ; d-$ энергоподвод $g_{2, \max }=g\left(r_{2}, M_{r 1, \max }\right)$ как функции $F_{Q}, 1-n=1,2-n=2$.

$H_{2}\left(F_{E}\right)$, приведенным на рис. $2, a$. Если в $F_{E}$-варианте как в цилиндрическом, так и в сферическом случае зависимости $H_{2}\left(F_{E}\right)$ слегка вогнуты (выпуклостью вниз), то в $F_{Q}$-варианте зависимости $H_{2}\left(F_{Q}\right)$ близки к линейным при $n=1$ и 2.

В $F_{Q}$-варианте получили близкое к нулевому значение $H_{2}\left(F_{Q}\right) \approx 0$ при $F_{Q 0}=-0.6967$ в сферическом источнике. Это значение больше, чем $F_{Q 0}=-0.5261$ в цилиндрическом источнике.

В противоположность этому в $F_{E}$-варианте нашли $F_{E 0}=-0.1200$ в сферическом источнике. Это значение меньше, чем $F_{E 0}=-0.2101$ в цилиндрическом случае.

\section{3. Зависимость энтальпии $\boldsymbol{H}_{2}$ в конце зоны от числа Маха $M_{r 1}, F_{Q}$-вариант}

Зафиксируем координату замыкающего сечения $r_{2}$ и будем варьировать число Маха на входе в зону $M_{r 1}$. Заметим, что координата $r_{1}$ входа в зону связана с числом Maхa $M_{r 1}$ (и температурой $T_{1}$ или любой другой газодинамической величиной $\rho_{1}, p_{1}, u_{1} \ldots$ на входе). Каждому сечению $r_{1}$ соответствуют два значения $M_{r 1}\left(T_{1}, \rho_{1}, \ldots\right)$ : дозвуковое $M_{r 1}<1$ и сверхзвуковое $M_{r 1}>1$. Приведенная выше постановка задачи допускает сколько угодно близкие значения к $M_{r 1}=1$ сверху $\left(M_{r 1}>1\right)$ или снизу $\left(M_{r 1}<1\right)$, исключая строго единичное значение $M_{r 1}$. Итоговые изменения полной энтальпии $H_{2}$ к концу зоны сопоставим при различных значениях силовых параметров $F_{Q}$ (рис. 3) и $F_{E}$ (рис. 4 ).

На рис. 3, $a$ приведены зависимости $H_{2}\left(M_{r 1}\right)$ при силовом параметре $F_{Q}=0,0.5,-0.3$, кривые $1-3$ для цилиндрического источника; на рис. $3, b$ - для сферического источника при силовом параметре $F_{Q}=0$, $0.5,-0.5$, кривые 4-6. На всех кривых есть локальные максимумы по $M_{r 1}: H_{2, \max }=3.427,8.146$, 0.976 при $M_{r 1, \max }=0.593,0.506,0.822$ (рис. 3, $a$, кривые $1-3, n=1), H_{2, \max }=17.234,30.796,4.522$ при $M_{r 1, \max }=0.441,0.411,0.551$ (рис. 3, $b$, кривые 4-6, $n=2)$. При неотрицательном силовом параметре $F_{Q} \geq 0$ в цилиндрическом источнике увеличение энтальпии в точке максимума составило от 3.427 до 8.146. В сферическом источнике увеличение энтальпии в приведенных примерах составило, исключая отрицательные значения силового параметра $F_{Q}$, от 17.2 до 30.8. Соответствую- 

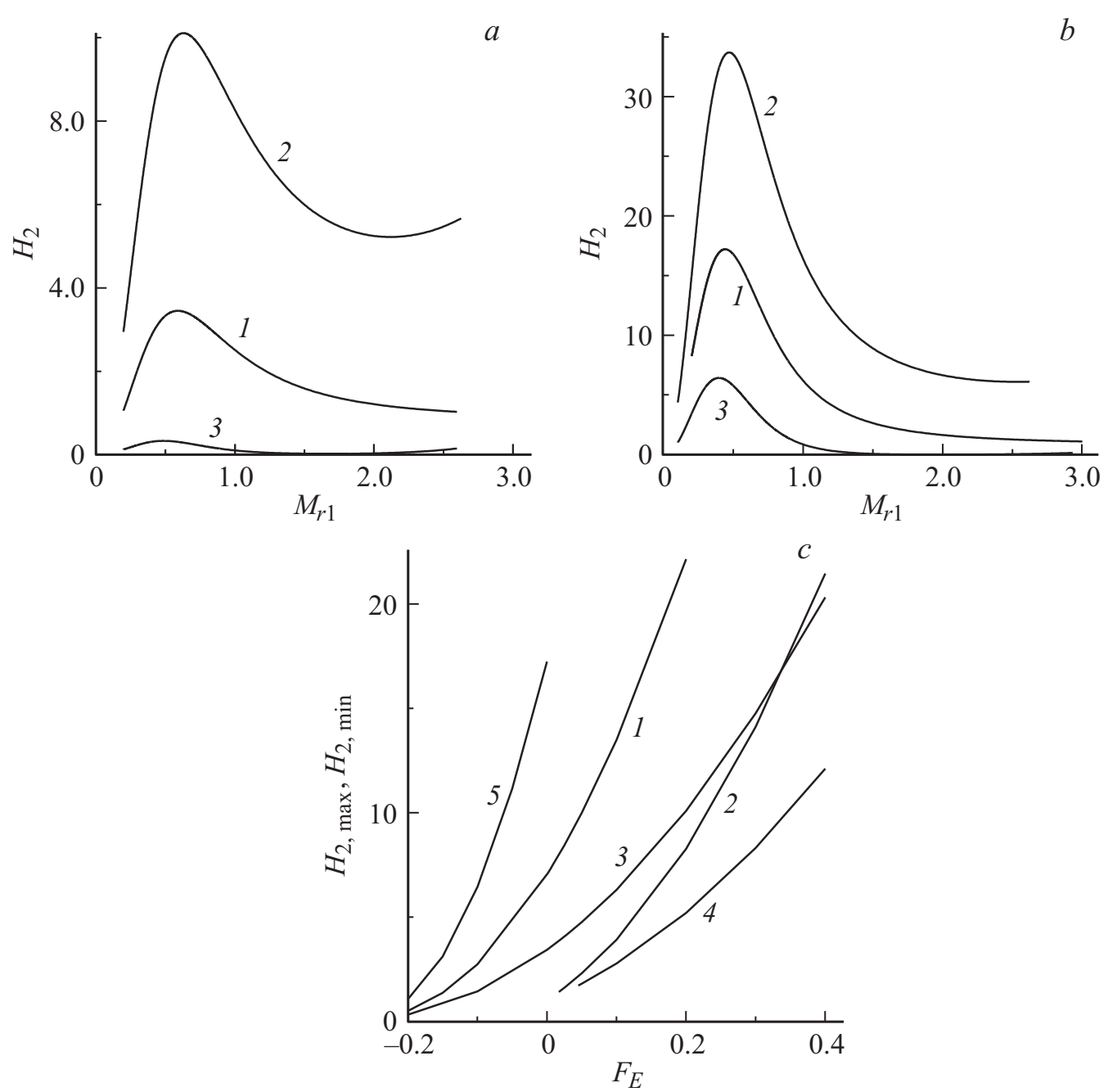

Рис. 4. $a-$ полная энтальпия $H_{2}$ на выходе $r_{2}=3$, в цилиндрическом источнике $n=1$ как функция числа Маха $M_{r 1} .1-$ силовой параметр $F_{E}=0,2-0.2,3--0.2 ; b-$ энтальпия $H_{2}\left(M_{r 1} ; r_{2}=3\right)$, сферический источник, $n=2: 1-F_{E}=0,2-0.1$, $3--0.1 ; c-$ максимальные и минимальные значения энтальпии в зависимости от $F_{E}: 1-H_{2, \max }, 2-H_{2, \min }, r_{2}=5,3-H_{2, \max }$, $4-H_{2, \min }, r_{2}=3, n=1 ; 5-H_{2, \max }, r_{2}=3, n=2$.

щие значения чисел Маха укладываются в узкий дозвуковой диапазон $M_{r 1, \max }=0.506-0.593$ (рис. $3, a, n=1$ ) и $M_{r 1, \max }=0.411-0.441$ (рис. $3, b, n=2$ ).

На рис. 3, $c$ построены зависимости максимальной энтальпии $H_{2, \max }$ от силового параметра $F_{Q}$ для цилиндрического источника (кривая 1) и сферического источника (кривая 2). В последнем случае значения $H_{2, \max }$ существенно больше. Зависимости $H_{2, \max }\left(F_{Q} ; M_{r 1, \max }\right)$ близки к линейным. На рис. $3, d$ приведены функции энергоподвода $g_{2, \max }\left(F_{Q} ; M_{r 1, \max }\right)$ в конце зоны, в точке максимума по $M_{r 1}$, в зависимости от силового параметра $F_{Q}$. Отметим, что из трех параметров (замыка-

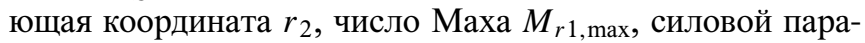
метр $F_{Q}$ ), определяющих увеличение энтальпии $H_{2}$, число Маха $M_{r 1, \max }$ представляется наиболее интересным и важным, поскольку силовой параметр и замыкающая координата с увеличением значения дают монотонный рост энтальпии, а вариации числа Маха - локальный сильно выраженный максимум.

\section{4. Зависимость энтальпии $\mathrm{H}_{2}$ в конце зоны от числа Маха $M_{r 1}, F_{E}$-вариант}

На рис. 4, $a, b$ представлены зависимости полной энтальпии $H_{2}\left(M_{r 1}\right)$ при трех значениях силового параметра $F_{E}=0$ (кривые 1$), 0.2(2),-0.2$ (3) в цилиндрическом источнике и $F_{E}=0(1), 0.1$ (2), - 0.1 (3) в сферическом. В отличие от аналогичных зависимостей $H_{2}\left(M_{r 1}\right)$, построенных при различных $F_{Q}$ на рис. $3, a, b$, в рассматриваемом $F_{E}$-варианте при достаточно больших значениях силового параметра $F_{E}$ и значениях замыкающей зону координаты $r_{2}$, кроме вышеупомянутого максимума $H_{2, \max }\left(F_{E} ; M_{r 1, \max }\right)$, в сверхзвуковой области имеются минимумы $H_{2, \min }\left(F_{E} ; M_{r 1, \min }\right)$ (рис. 4, $a$, кривая 2), $H_{2, \min }=5.20$ при $M_{r 1, \min }=2.12, F_{E}=0.2$, $r_{2}=3$.

На рис. 4, $c$ показаны в зависимости от силового параметра $F_{E}$ максимальные энтальпии $H_{2, \max }-$ кривая 1 , минимальные $H_{2, \min }-2, r_{2}=5, n=1$, а также 

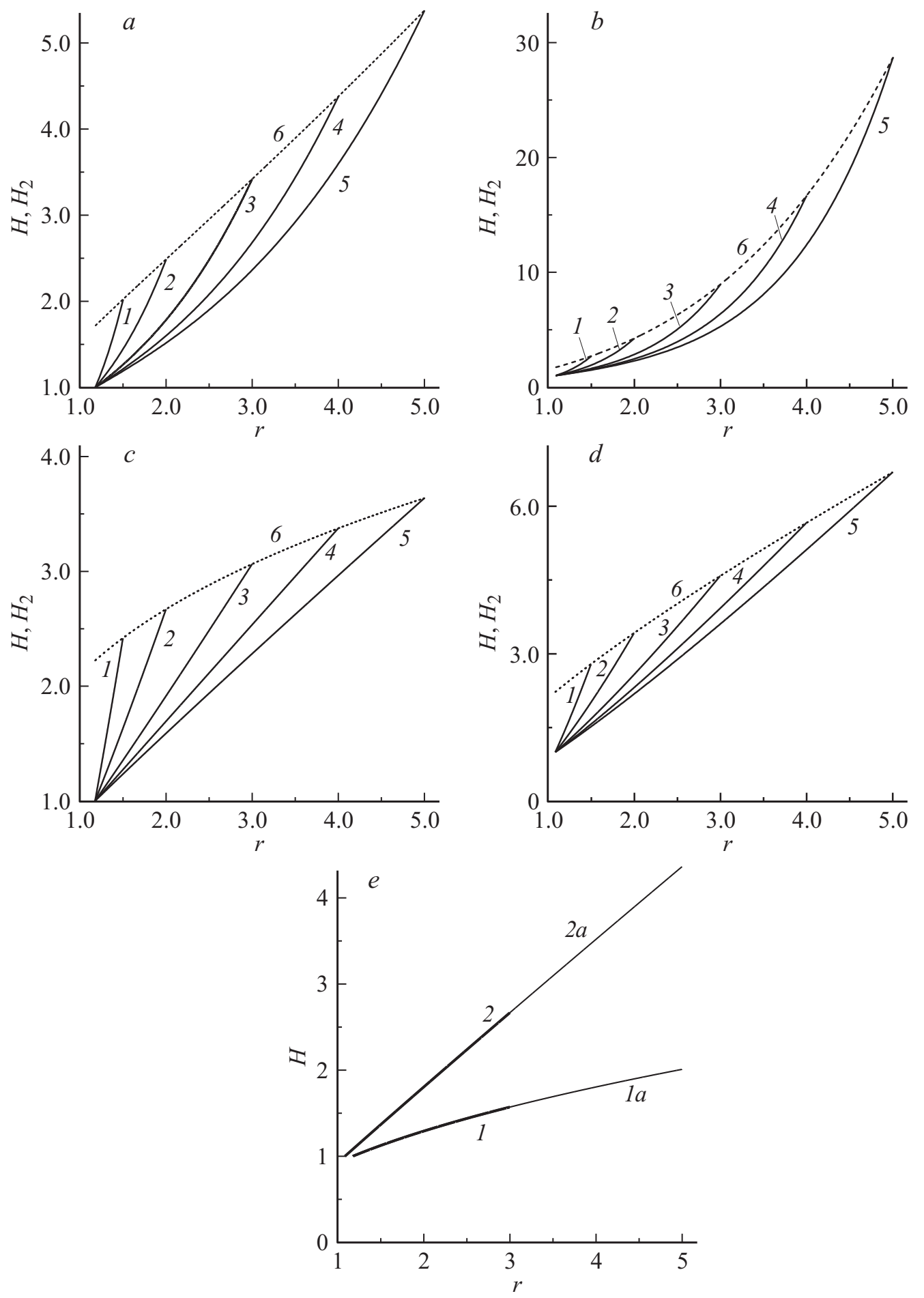

Рис. 5. $a$ - энтальпия $H(r)$ внутри зоны, кривые $1-5\left(r_{2}=1.5,2,3,4,5\right)$ и в конце $H_{2}=H\left(r_{2}\right)$, огибающая $6, n=1$, число Маха $M_{r 1}=1.5$, силовой параметр $F_{E}=0.1 ; b-$ то же самое при $n=2, M_{r 1}=1.5, F_{E}=0.1 ; c$ - то же самое при $n=1, M_{r 1}=1.5$, $F_{Q}=0.5 ; d-$ при $n=2, M_{r 1}=1.5, F_{Q}=0.5 ; e-F_{Q}=0=F_{E}, n=1: 1-r_{2}=3,1 a-5, n=2,2-r_{2}=3,2 a-5$.

$H_{2, \max }-3, H_{2, \min }-4, r_{2}=3, n=1$, цилиндрический источник, $H_{2, \max }-5, r_{2}=3, n=2$, сферический. В сферическом источнике минимумы по числам Маха $M_{r 1}$ не обнаружены. Соответствующая максимумам кривая 5 , $H_{2, \max }\left(F_{E}, M_{r 1, \max }, r_{2}=3\right), n=2$ нарастает с увеличением $F_{E}$ быстрее кривых 1 и $3\left(r_{2}=5,3 ; n=1\right)$.
Если сопоставить рост максимальных энтальпий $H_{2, \max }\left(F_{E}, r_{2}=3\right)$ с увеличением силового параметpa $F_{E}$, кривые 3 и 5 на рис. $4, c$, с аналогичными зависимостями $H_{2, \max }\left(F_{Q}, r_{2}=3\right)$ от силового параметpa $F_{Q}$, показанными на рис. $3, c$, заметим, что при $F_{Q}=0.5$ величина $H_{2, \max }$ составила $8.146(n=1)$ и 30.79 
$(n=2)$. При $F_{E}=0.2$ получили $H_{2, \max } \approx 10.08(n=1)$ и $56.07(n=2)$. При этом в $F_{E}$-варианте сила равна $F_{2, \max }=0.1082(n=1), 0.1089(n=2) . \mathrm{B} F_{Q}$-варианте сила равна $F_{2, \max }=0.04094(n=1), 0.00817(n=2)$.

Зависимости значений внешней силы $F_{2, \max }$, соответствующей максимумам, от силового параметра $F_{E}$ для цилиндрического и сферического источников практически не различаются в диапазоне $[-0.2,0.2]$ и близки к линейным.

Сопоставление величин энергоподвода $g_{2, \max }$ (не приведены), соответствующих максимумам, от силового параметра $F_{E}$ показало отличие от соответствующих зависимостей $g_{2, \max }\left(F_{Q}\right)$ (рис. $3, d$, кривые 1,2 ) слабым нарастанием темпа увеличения с ростом $F_{E}$, в то время как зависимости $g_{2, \max }\left(F_{Q}\right)$ близки к линейным. Зависимости $g_{2, \max }\left(F_{E}\right)$ нарастают круче, например, при $n=2$ величина $g_{2, \max }\left(F_{E}=0.2\right)=2.39$, а $g_{2, \max }\left(F_{Q}=0.5\right)=0.994 ;$ при $n=1 \quad g_{2, \max }\left(F_{E}=0.2\right)=$ $=0.731$, a $g_{2, \max }\left(F_{Q}=0.5\right)=0.459$.

\section{5. Зависимость энтальпии $\mathrm{H}_{2}$ от величины замыкающей координаты $r_{2}$}

Величина замыкающей зону координаты $r_{2}$ (или протяженность зоны) уже упоминалась среди факторов, влияющих на приращение энтальпии $\mathrm{H}_{2}$ вследствие комбинированного энергосилового воздействия.

На рис. 5, $a(n=1)$ построены распределения энтальпии $H(r)$ внутри зоны, кривые $1-5\left(r_{2}=1.5,2,3,4,5\right)$ при $M_{r 1}=1.5, F_{E}=0.1$. Огибающая 6 , которая описывает энтальпию в конце зоны $H_{2}=H\left(r_{2}\right)$, близка к линейной функции. Увеличение энтальпии $H_{2}$ при $r_{2}=3$ составило 3.42 по сравнению с начальным значением $H_{1}=1$; при $r_{2}=5$ - величина $H_{2}=5.37$.

В сферическом источнике $(n=2$, рис. $5, b)$ при силовом параметре $F_{E}=0.1$, числе Маха $M_{r 1}=1.5$ и замыкающей зону координате $r_{2} \geq 3$ установлено увеличение энтальпии $\mathrm{H}_{2}$ более чем на порядок по сравнению с начальным значением. Огибающая 6 вогнутая (выпуклостью вниз), что означает увеличение темпа прироста энтальпии $H_{2}$ с ростом $r_{2}$.

На рис. $5, c, d$ рассматриваются $F_{Q}$-варианты при $F_{Q}=0.5, M_{r 1}=1.5, n=1$ (рис. $\left.5, c\right)$ и $n=2$ (рис. $\left.5, d\right)$. Зависимости для распределений энтальпии $H(r)$ внутри зоны воздействия близки к линейным. Огибающая $\mathrm{H}_{2}$ (кривая 6) выпуклая как в цилиндрическом $(n=1)$, так и в сферическом $(n=2)$ вариантах. Количественно увеличение энтальпии $\mathrm{H}_{2}$ в сферическом источнике приблизительно вдвое превышает соответствующие значения в цилиндрическом источнике при $r_{2}=5$ и выше.

На рис. 5,e приведены зависимости энтальпии $H(r)$ при $r_{2}=3$ (полужирные кривые 1 и $2, n=1$ и 2) и при $r_{2}=5$ (простые кривые $1, a$ и $2, a, n=1$ и 2). Кривые $H(r)$ с бо́льшими значениями координаты замыкающего сечения $r_{2}=5$ на участке от $r=r_{1}$ до $r=r_{2}=3$ совпадают с зависимостями с меньшими $r_{2}$, например $r_{2}=1.5,2,3$, как показано на рис. 5, e. Такова отличительная характерная черта базового варианта $F(r)=0$ от комбинированных случаев воздействия внешней силой и энергоподводом во всех рассмотренных вариантах.

\section{Выводы}

1. При комбинированном воздействии в режиме постоянного числа Маха $M_{r 1}$ при неотрицательных значениях силовых параметров $F_{Q}, F_{E} \geq 0$ энтальпия, температура, скорость монотонно растут по координате, как в базовом энергетическом варианте.

2. Энтальпия $H_{2}$ в конце зоны воздействия увеличивается с нарастанием темпа при увеличении силового параметра $F_{E}$ (сила задана на единицу объема) и линейно с ростом $F_{Q}$ (сила задана на единицу массы) в цилиндрическом и сферическом источнике.

3. При отрицательных $F_{Q}, F_{E}$ в пределе энтальпии $H_{2}$, близкой к нулю, функция энергообмена $g(r)$, поддерживающая число Маха постоянным, отрицательна по всей зоне в цилиндрическом источнике (охлаждение), либо во второй половине зоны в сферическом источнике.

4. Обнаружены локальные максимумы энтальпии $H_{2, \max }$ в дозвуковом диапазоне чисел Маха $M_{r 1}$ в цилиндрическом и сферическом источнике, в $F_{Q^{-}}, F_{E}$-вариантах. Пики $H_{2, \max }$ существенно выше в сферическом источнике.

5. Энтальпия за зоной $H_{2}$ монотонно растет с увеличением замыкающей координаты $r_{2}$ в цилиндрическом источнике и в сферическом источнике, в $F_{Q^{-}}$и $F_{E}$-вариантах.

6. В базовом энергетическом случае $F_{Q}=0=F_{E}$ зависимости энтальпии $H(r), H_{2}$ от координаты $r$ и от координаты замыкающего сечения $r_{2}$ ложатся на одну кривую при возрастании $r_{2}$ как в цилиндрическом источнике, так и в сферическом.

\section{Список литературы}

[1] Курант Р., Фридрихс К. Сверхзвуковые течения и ударные волны. М.: ИЛ, 1950. 426 с. (Courant R., Friedrichs K.O. Supersonic Flow and Shock Waves. NY.: Intersience, 1948).

[2] Мизес $P$. Математическая теория течений сжимаемой жидкости. М.: ИЛ, 1961. 588 с. (Mises R. Mathematical Theory of Compressible Fluid Flow. NY.: Academic Press INC Publishers, 1958).

[3] Черный Г.Г. Газовая динамика. М.: Наука, 1988. 424 с.

[4] Абрамович Г.Н. // ДАН СССР. 1946. Т. 54. № 7. C. $579-581$.

[5] Вулис Л.А. // ДАН СССР. 1946. Т. 54. № 8. С. 669-672.

[6] Лойцянский Л.Г. Механика жидкости и газа. М.: Наука, 1973. $848 \mathrm{c.}$

[7] Абрамович Г.Н. Прикладная газовая динамика. Ч. 1. М.: Наука, 1991. 600 с.

[8] Кучеров А.Н. // ИФЖ. 2014. Т. 87. № 1. С. 129-138.

[9] Кучеров А.Н. // ЖТФ. 2015. Т. 85. Вып. 10. С. 34-41.

[10] Кучеров А.Н. Основные закономерности теплового запирания цилиндрического массового вихреисточника (вихрестока). Препринт № 158. М.: Изд. отд. ЦАГИ, 2010. 40 с. 
[11] Плотность, энтальпия, энтропия и изобарная теплоемкость жидкого и газообразного азота при температурах $70-1500 \mathrm{~K}$ и давлениях 0.1-100 МПа. ГСССД 4-78. М.: Изд. стандартов, 1978. $12 \mathrm{c.}$

[12] Кислород жидкий и газообразный, плотность, энтальпия, энтропия и изобарная теплоемкость при температурах $70-1000 \mathrm{~K}$ и давлениях 0.1-100 МПа. ГСССД 19-81. М.: Изд. стандартов, 1982. $11 \mathrm{c.}$

[13] Воздух жидкий и газообразный. Плотность, энтальпия, энтропия и изобарная теплоемкость при температурах $70-1500 \mathrm{~K}$ и давлениях 0.1-100 МПа. ГСССД 8-79, c. 19-34. В сб.: Свойства материалов и веществ. Воздух и его основные компоненты. Вып. 2. Таблицы стандартных справочных данных. М.: Гос. ком. по управлению качеством продукции и стандартам, 1991. 128 с. 